\title{
Strange nonchaotic attractors in Harper maps
}

\author{
Àlex Haro \\ Departament de Matemàtica Aplicada i Anàlisi, Universitat de Barcelona, Gran Via 585, \\ Barcelona 08007, Spain \\ Joaquim Puig \\ Departament de Matemàtica Aplicada I, Universitat Politècnica de Catalunya, Diagonal 647, \\ Barcelona 08028, Spain
}

(Received 24 April 2006; accepted 8 July 2006; published online 27 September 2006)

\begin{abstract}
We study the existence of strange nonchaotic attractors (SNA) in the family of Harper maps. We prove that for a set of parameters of positive measure, the map possesses a SNA. However, the set is nowhere dense. By changing the parameter arbitrarily small amounts, the attractor is a smooth curve and not a SNA. (C) 2006 American Institute of Physics. [DOI: 10.1063/1.2259821]
\end{abstract}

In the last two decades there has been an enormous interest in the so-called strange nonchaotic attractors (SNA). These attracting invariant objects of dynamical systems capture the evolution of a large subset of the phase space and are very relevant for their description. In particular, SNA are geometrically complicated (they are strange) and their dynamics is regular (in most of the examples, quasiperiodic). SNA are typically observed in systems where there is a coupling between different dynamics. In contrast to the vast amount of numerical and experimental work in the area, there are only few rigorous proofs. The goal of this paper is to prove the existence and abundance of SNA in the family of Harper maps. This family arises from a model in mathematical physics and it is a paradigm of a 1D quasiperiodically forced map. Our approach connects the spectral theory of Schrödinger operators and the theory of nonuniformly hyperbolic systems. So, even if the proof is made for a concrete family, many of the arguments apply to other families.

\section{INTRODUCTION}

The study of the attractors of a dynamical system is a topic of great interest, because these invariant sets capture the asymptotic behavior of the system. It has been known for a long time that attractors can be strange, ${ }^{1}$ i.e., geometrically complicated. The first examples of strange attractors were chaotic, i.e., with sensitive dependence on initial conditions. ${ }^{2}$ In many physically relevant situations, one has to consider systems subject to external forcing, which may depend on several frequencies in a quasiperiodic way. In such dissipative systems, one expects attractors which retain part of the quasiperiodicity. This was observed in Ref. 3 where strange attractors that are nonchaotic were found, and it has stimulated much numerical experimentation (see the review in Ref. 4) as well as rigorous analysis of some particular models. ${ }^{5-9}$

Our goal in this paper is to prove the existence and abundance of strange nonchaotic attractors (SNA) in the family of Harper maps. This is a family of 1D quasiperiodically forced maps that many authors have suggested, based on numerical experiments and heuristic arguments, as a scenario in which SNA appear, ${ }^{10-15}$ but proofs are scarce. This equation also arises naturally from problems in mathematical physics and it is a paradigm of a 1D quasiperiodically forced map.

In plain words, by SNA we mean in this paper an invariant set which is the graph of a measurable and nowhere continuous function (it is strange), that carries a quasiperiodic dynamics (it is nonchaotic) and it attracts exponentially fast almost every orbit in phase space (it is an attractor). A more precise definition will be given later, see Definition 1 .

We prove that these SNA are typical but not robust in the family of Harper maps, in the sense that they exist for a positive measure Cantor set of the parameter space. That is to say, SNA are abundant, but an arbitrarily small perturbation can make them continuous (in fact analytic) attracting invariant curves.

In our analysis, we exploit the connections between (a) the dynamical properties of the Harper map (a 1D quasiperiodically forced map); (b) the spectral properties of the Harper operator (an example of a quasiperiodic Schrödinger operator); (c) the geometrical properties of the Harper linear skew product (a 2D quasiperiodically forced linear map).

In recent years our knowledge of the spectral properties of the Harper operator, also known as the almost Mathieu operator, and related quasiperiodic Schrödinger operators has advanced spectacularly.

In particular, the connections between (b) and (c) have been successfully applied to solve the "Ten Martini Problem"16-18 on the Cantor structure of the spectrum of the Harper operator. The connection between (a) and (c) in similar models has been used to study the linearized dynamics around invariant tori in quasiperiodic systems. ${ }^{19}$ Specifically, the formation of SNA in this linearized dynamics is suggested to be a mechanism of breakdown of invariant tori. ${ }^{20}$

Even if we deal with one-dimensional quasiperiodically forced systems, many of the techniques that we use in this paper also apply to higher dimensional or less regular settings, including other types of dynamics on the external forcing. 
A consequence of our approach is that neither the arithmetic properties of the frequency of the quasiperiodic forcing nor the localization properties of the Harper operator are crucial for the existence of SNA. These two hypotheses lie at the heart of many heuristic arguments for establishing the existence of SNA in Harper maps. ${ }^{10,11,14,21,22}$

\section{HARPER MAPS, COCYCLES, AND OPERATORS}

The family of quasiperiodically forced dynamical systems under investigation in this paper is the family of Harper maps

$$
\begin{aligned}
y_{n+1} & =\underbrace{\frac{1}{a-b \cos \left(2 \pi \theta_{n}\right)-y_{n}},}_{f_{a, b}\left(y_{n}, \theta_{n}\right)} \\
\theta_{n+1} & =\theta_{n}+\omega \quad(\bmod 1),
\end{aligned}
$$

where $y \in \bar{R}=[-\infty,+\infty]$ and $\theta \in \mathbb{T}=\mathbb{R} / \mathbb{Z}$ are the phase space variables, $a, b$ are the parameters, and $\omega$ is the frequency (it is assumed to be irrational). Notice that a Harper map is a skew product map $F_{a, b, \omega}\left(y_{n}, \theta_{n}\right)=\left(f_{a, b}\left(y_{n}, \theta_{n}\right), \theta_{n}+\omega\right)$, defining a dynamical system in $\bar{R} \times \mathrm{T}$ whose evolution from an initial condition $\left(y_{0}, \theta_{0}\right)$ is described by the $N$ th power $F_{a, b, \omega}^{(N)}\left(y_{0}, \theta_{0}\right)=\left(f_{a, b}^{(N)}\left(y_{0}, \theta_{0}\right), \theta_{0}+N \omega\right)$, for $N \in \mathbb{Z}$.

In a Harper map the parameter $a$ is called the energy or the spectral parameter because after writing $y_{n}=x_{n-1} / x_{n}$ this family is equivalent to the family of Harper equations, which are second-order difference equations

$$
x_{n+1}+x_{n-1}+b \cos \left(2 \pi\left(\theta_{0}+n \omega\right)\right) x_{n}=a x_{n} .
$$

These equations are physically relevant because they show up as eigenvalue equations of Harper operators (also known as almost Mathieu operators),

$$
\left(H_{b, \omega, \theta_{0}} x\right)_{n}=x_{n+1}+x_{n-1}+b \cos \left(2 \pi\left(\theta_{0}+n \omega\right)\right) x_{n} .
$$

These are bounded and self-adjoint operators on $\ell^{2}(Z)$ whose spectrum $\Sigma_{b, \omega}$, that does not depend on $\theta_{0}$, describes the energy spectrum of an electron in a rectangular lattice subject to a perpendicular magnetic flux. ${ }^{23,24}$ Their study is relevant for the explanation of phenomena such as the quantum Hall effect. ${ }^{25}$

The formulation of the second-order difference equation (2) as a first-order system is the Harper linear skew product

$$
\begin{aligned}
& \underbrace{\left(\begin{array}{c}
x_{n+1} \\
x_{n}
\end{array}\right)}_{v_{n+1}}=\underbrace{\left(\begin{array}{cc}
a-b \cos \left(2 \pi \theta_{n}\right) & -1 \\
1 & 0
\end{array}\right)}_{M_{a, b}\left(\theta_{n}\right)} \underbrace{\left(\begin{array}{c}
x_{n} \\
x_{n-1}
\end{array}\right)}_{v_{n}}, \\
& \theta_{n+1}=\theta_{n}+\omega \quad(\bmod 1),
\end{aligned}
$$

whose evolution is given by the Harper cocycle

$$
M_{a, b, \omega}^{(N)}\left(\theta_{0}\right)= \begin{cases}M_{a, b}\left(\theta_{N-1}\right) \ldots M_{a, b}\left(\theta_{0}\right) & \text { if } N>0, \\ I \quad \text { if } N=0, & \\ M_{a, b}^{-1}\left(\theta_{N}\right) \ldots M_{a, b}^{-1}\left(\theta_{-1}\right) & \text { if } N<0 .\end{cases}
$$

Note that (1) describes the evolution of the slope $y_{n}$ of vectors $v_{n}$ evolving under the action of the linear skew prod- uct (4). That is, (1) is the projectivization of (4). In polar coordinates, $\varphi=\arctan y \in \mathrm{P} \simeq[-\pi / 2, \pi / 2]$, this projectivization becomes

$$
\begin{aligned}
& \varphi_{n+1}=\underbrace{\arctan \left(\frac{1}{a-b \cos \left(2 \pi \theta_{n}\right)-\tan \varphi_{n}}\right)}_{\tilde{f}_{a, b}\left(\varphi_{n}, \theta_{n}\right)}, \\
& \theta_{n+1}=\theta_{n}+\omega \quad(\bmod 1) .
\end{aligned}
$$

This polar Harper map is a skew product map $\widetilde{F}_{a, b, \omega}\left(\varphi_{n}, \theta_{n}\right)=\left(\widetilde{f}_{a, b}\left(\varphi_{n}, \theta_{n}\right), \theta_{n}+\omega\right)$, defining a dynamical system in $\mathrm{P} \times \mathrm{T}$ whose evolution from an initial condition $\left(\varphi_{0}, \theta_{0}\right)$ is described by the $N$ th power $\tilde{F}_{a, b, \omega}^{(N)}\left(\varphi_{0}, \theta_{0}\right)=\left(\tilde{f}_{a, b}^{(N)}\left(\varphi_{0}, \theta_{0}\right), \theta_{0}+N \omega\right)$, for $N \in \mathbb{Z}$. The projective and the polar formulations are equivalent, but we will mostly use the polar one for simplicity.

\section{EXISTENCE AND ABUNDANCE OF SNA IN HARPER MAPS}

In this section we establish the main result of this paper, on existence and abundance of SNA in Harper maps. Let us start with some generalities.

A (continuous, smooth, analytic) skew product map in $\mathrm{P} \times \mathrm{T}$ of the form

$$
\varphi_{n+1}=\tilde{f}\left(\varphi_{n}, \theta_{n}\right), \quad \theta_{n+1}=\theta_{n}+\omega \quad(\bmod 1),
$$

where $\omega$ is irrational, defines a quasiperiodically forced system in $\mathrm{P}$. In both $\mathbb{T}$ and $\mathrm{P}$ we consider the Lebesgue measure, and in $\mathrm{P} \times \mathrm{T}$ the corresponding product measure.

In this setting, we consider the following working definition of SNA. We emphasize that the definition itself of SNA is a subject of much debate and the definition given here may not be suitable for other models.

Definition 1: Let $\Phi \subset \mathbb{P} \times \mathbb{T}$ be a subset of the phase space of the form

$$
\Phi=\{(\varphi(\theta), \theta), \theta \in \Theta\}
$$

where $\Theta \subset \mathbb{T}$ is a full measure set and $\varphi: \Theta \rightarrow P$ is a measurable function. That is, $\Phi$ is the graph of a measurable function. Then

- We say that $\Phi$ is strange if $\varphi$ cannot be extended to a graph of a continuous function in $T$.

- We say that $\Phi$ is invariant under (7) if for all $\theta \in \Theta$ we have $\theta+\omega \in \Theta$ and $\tilde{f}(\varphi(\theta), \theta)=\varphi(\theta+\omega)$. Moreover, $\Phi$ is a nonchaotic invariant set because its dynamics is quasiperiodic.

- We say that $\Phi$ is an attractor of (7) if it is invariant and for almost all initial condition $(\varphi, \theta) \in \mathbb{P} \times \mathbb{T}$,

$$
\lim _{N \rightarrow+\infty}\left|\widetilde{f}_{a, b, \omega}^{(N)}(\varphi, \theta)-\varphi\left(\theta_{N}\right)\right|_{\mathrm{P}}=0,
$$

where $|\cdot|_{\mathrm{P}}$ denotes the distance of two angles in $\mathbb{P}$.

We say that $\Phi$ is a strange nonchaotic attractor if $\Phi$ satisfies the three properties above.

The main result of this paper is 
Main Theorem: For $|b|>2$, the Harper map (6) has a SNA for all the values $a$ in a Cantor set of measure $|4-2| b||>0$.

\section{PROOF OF THE MAIN THEOREM}

In this section we prove the Main Theorem. The proof is based on the relation among the hyperbolicity properties of Harper linear skew products (4), the dynamics of (polar) Harper maps (6) and the spectral properties of Harper operators (3).

\section{A. Lyapunov exponents and hyperbolicity}

To understand the dynamics of Harper linear skew products it is important to know the growth properties of the solutions. The exponential growth is measured by the Lyapunov exponents which we now define. Given any nontrivial initial condition of the skew product $(4),\left(v_{0}, \theta_{0}\right)$ with $v_{0} \neq 0$, the (forward) Lyapunov exponent for $\left(v_{0}, \theta_{0}\right)$ is the limit

$\lambda_{a, b, \omega}\left(v_{0}, \theta_{0}\right)=\lim _{N \rightarrow+\infty} \frac{1}{N} \log \left|v_{N}\right|=\lim _{N \rightarrow+\infty} \frac{1}{N} \log \left|M_{a, b, \omega}^{(N)}\left(\theta_{0}\right) v_{0}\right|$

whenever the limit exists (in which case it is finite).

If $v_{0}=\left(x_{-1}, x_{0}\right)$ and $\varphi_{0}=\arctan \left(x_{-1} / x_{0}\right)$, then one can also define the (forward) Lyapunov exponent of the Harper map (6) for the initial condition $\left(\varphi_{0}, \theta_{0}\right)$ by

$$
\begin{aligned}
\beta_{a, b, \omega}\left(\varphi_{0}, \theta_{0}\right) & =\lim _{N \rightarrow+\infty} \frac{1}{N} \log \left|\frac{\partial \varphi_{N}}{\partial \varphi_{0}}\left(\varphi_{0}, \theta_{0}\right)\right| \\
& =\lim _{N \rightarrow+\infty} \frac{1}{N} \log \left|m_{a, b, \omega}^{(N)}\left(\varphi_{0}, \theta_{0}\right)\right|
\end{aligned}
$$

where

$$
m_{a, b, \omega}^{(N)}\left(\varphi_{0}, \theta_{0}\right)=\frac{\partial \tilde{f}_{a, b}}{\partial \varphi}\left(\varphi_{N-1}, \theta_{N-1}\right) \ldots \frac{\partial \tilde{f}_{a, b}}{\partial \varphi}\left(\varphi_{0}, \theta_{0}\right) .
$$

An easy computation shows the relation

$$
\beta_{a, b, \omega}\left(\varphi_{0}, \theta_{0}\right)=-2 \lambda_{a, b, \omega}\left(v_{0}, \theta_{0}\right) .
$$

Backward Lyapunov exponents are defined by replacing $\lim _{N \rightarrow+\infty}$ with $\lim _{N \rightarrow-\infty}$ in the above formulation.

Oseledec ${ }^{26}$ showed that for almost every initial condition $\left(v_{0}, \theta_{0}\right)$ the Lyapunov exponent exists and it is almost everywhere a constant value. This value is precisely the averaged Lyapunov exponent

$$
\bar{\lambda}_{a, b, \omega}=\lim _{N \rightarrow+\infty} \frac{1}{N} \int_{\mathbb{T}} \log \left|M_{a, b, \omega}^{(N)}(\theta)\right| d \theta,
$$

which is non-negative and exists by Kingman's subadditive ergodic theorem. ${ }^{27}$

The case of the nonzero averaged Lyapunov exponent $(\bar{\lambda}>0)$ which we call hyperbolic, is important for our purposes. In this case, there exists a full measure set $\Theta \subset \mathbb{T}$ such that for every $\theta \in \Theta$ one has a splitting

$$
\mathbb{R}^{2}=W^{s}(\theta) \oplus W^{u}(\theta)
$$

characterized by, for $v \neq 0$,

$$
v \in W^{S}(\theta) \Leftrightarrow \lim _{N \rightarrow \pm \infty} \frac{1}{N} \log \left|M^{(N)}(\theta) v\right|=-\bar{\lambda}
$$

and

$$
v \in W^{u}(\theta) \Leftrightarrow \lim _{N \rightarrow \pm \infty} \frac{1}{N} \log \left|M^{(N)}(\theta) v\right|=+\bar{\lambda} .
$$

$W^{s}(\theta)$ and $W^{u}(\theta)$ are the stable and unstable subspaces at $\theta$, respectively. ${ }^{26}$ The elements of the set $\Theta$ are referred to as the Lyapunov regular points.

In the phase space $R^{2} \times T$, one can form the product sets $W^{s}$ and $W^{u}$ whose elements are pairs $(v, \theta)$ with $v \in W^{s}(\theta)$ and $W^{u}(\theta)$ respectively (whenever these subspaces are defined). These are the stable and unstable subbundles. By Oseledec $^{26}$ the $\theta$ dependence of the decomposition is measurable but not necessarily continuous.

\section{B. Nonuniform hyperbolicity and SNA}

The property of hyperbolicity of the Harper cocycle (4) transfers to the dynamics of the Harper map (6), which reflects how the linear skew product (4) changes directions of vectors. So, if we define $\varphi^{s}(\theta)$ and $\varphi^{u}(\theta)$ as the angles of the subbundles $W^{s}(\theta)$ and $W^{u}(\theta)$, respectively, that is, they are the elements of $\mathbb{P}$ such $\left(\cos \varphi^{s}(\theta), \sin \varphi^{s}(\theta)\right)^{T}$ and $\left(\cos \varphi^{u}(\theta), \sin \varphi^{u}(\theta)\right)^{T}$ belong to $W^{s}(\theta)$ and $W^{u}(\theta)$, respectively, the product sets

$$
\Phi^{s}=\left\{\left(\varphi^{s}(\theta), \theta\right), \theta \in \Theta\right\} \text { and } \Phi^{u}=\left\{\left(\varphi^{u}(\theta), \theta\right), \theta \in \Theta\right\}
$$

are invariant under the Harper map and have quasiperiodic dynamics. Thus $\Phi^{s}$ and $\Phi^{u}$ are nonchaotic invariant sets.

Moreover, the decomposition of $\mathbb{R}^{2}$ into the direct sum of $W^{s}(\theta)$ and $W^{u}(\theta)$, for $\theta \in \Theta$, implies that every initial condition $(v, \theta)$ not lying on the stable subbundle is attracted to the unstable subbundle and grows exponentially in norm under the evolution given by (4). Looking at directions (which is what the polar Harper map (6) retains), forward orbits with initial condition $(\varphi, \theta)$ (other than $\left.\left(\varphi^{S}(\theta), \theta\right)\right)$ are exponentially attracted to $\Phi^{u}$, that is

$$
\beta(\varphi, \theta)=\lim _{N \rightarrow+\infty} \frac{1}{N} \log \left|\tilde{f}_{a, b, \omega}^{(N)}(\varphi, \theta)-\varphi^{u}\left(\theta_{N}\right)\right|_{\mathrm{P}}=-2 \bar{\lambda}<0,
$$

while backward orbits other than $\left.\left(\varphi^{u}(\theta), \theta\right)\right)$ are exponentially attracted to $\Phi^{s}$. Thus $\Phi^{u}$ is a nonchaotic attractor for the Harper map: for almost every initial condition, orbits are exponentially attracted to it. Similarly $\Phi^{s}$ is a nonchaotic repellor.

Remark 1: The notation $\Phi^{u}$ for an attractor and $\Phi^{s}$ for a repellor may look paradoxical, but it is kept for consistency. $\Phi^{u}$ is the projectivization of $W^{u}$ and $\Phi^{s}$ is the projectivization of $W^{s}$.

The regularity properties of the invariant subbundles $W^{s}, W^{u}$ of the Harper linear skew product are inherited by the invariant curves $\Phi^{s}, \Phi^{u}$ of the Harper map. 
When the splitting (12) is defined for all $\theta$, that is $\Theta$ $=\mathrm{T}$, the linear skew product is said to be uniformly hyperbolic and the subbundles are continuous. ${ }^{28,29}$ In such a case, the projectivizations $\Phi^{s}, \Phi^{u}$ are continuous invariant curves of the Harper map.

Remark 2: Johnson and Sell ${ }^{30,31}$ prove that in the uniformly hyperbolic case the stable and unstable subbundles are as smooth as the original system, and so are their projectivizations. See also Refs. 32 and 33. This follows from the fact that the dynamics on the base torus is a rotation. In the case of a Harper linear skew product, when it is uniformly hyperbolic, the stable and unstable subbundles are analytic. As a result, the projectivizations of the stable and the unstable subbundles are analytic invariant curves of the Harper map.

In contrast, if the skew product is nonuniformly hyperbolic, then the invariant subbundles are measurable but not continuous and their projectivizations $\Phi^{u}$ and $\Phi^{s}$ are measurable but not continuous functions of $\theta$. Moreover, discontinuities are propagated by the quasiperiodic dynamics and the invariance property of the attractor: if $\varphi^{u}$ is discontinuous at a single $\theta_{0}$ then the same happens for $\theta_{N}=\theta_{0}+N \omega$ for all $N$ $\in \mathbb{Z}$, so that the function $\varphi^{u}$ is nowhere continuous. The same result holds for $\varphi^{s}$.

In summary, when the skew product (4) is nonuniformly hyperbolic, $\Phi^{u}$ is a strange nonchaotic attractor (SNA) of the Harper map (6), see Definition 1. Moreover, almost every orbit in phase space is attracted to $\Phi^{u}$ at an exponential rate.

Remark 3: In principle one could look for SNA without exponential rate of attraction (for instance given by a power law). A candidate for such objects would be a Harper map at critical coupling $b=2$ and energies in the spectrum, as discussed in Ref. 34. For definiteness, we focus on the exponential case.

\section{SNA and spectrum}

In the Harper map, we can determine whether hyperbolicity is uniform or not by looking at the spectral problem of (3). Indeed, an energy $a$ is in the spectrum of the Harper operator (3) if, and only if, the corresponding linear skew product (4) is not uniformly hyperbolic. . $^{35,36}$

We will use an implication of this result if $a$ is in the spectrum of the Harper operator and the averaged Lyapunov exponent is nonzero at $a$, then the linear skew product is nonuniformly hyperbolic.

This is the key point in our approach and it is based in the following dichotomy for the linear quasiperiodic skew product: $^{28,29}$ either the skew product has a nontrivial bounded solution for some $\theta$ or the invariant subbundles exist for all $\theta$ and are as regular as the system.

The existence of nonuniformly hyperbolic linear skew products was already shown by Herman, ${ }^{37}$ who proved that

$$
\bar{\lambda}_{a, b, \omega} \geqslant \max \left(0, \log \frac{|b|}{2}\right)
$$

as long as $\omega$ is irrational. Moreover, Bourgain and Jitomirskaya ${ }^{38}$ proved that the equality in (16) holds if, and only if, $a$ is in the spectrum of the almost Mathieu operator.
Therefore, we have shown that for $|b|>2$ and $\omega$ irrational, a Harper map has a SNA for every value $a$ in the spectrum $\Sigma_{b, \omega}$ of the Harper operator (3), which is not empty (see next section).

In fact, we have even seen the following alternative: in the Harper map with $|b|>2$ and irrational frequency, either there is a SNA or an attracting analytic invariant curve. These SNA provide a rich class of examples where many properties conjectured for SNA can be rigorously proved.

\section{The last step: Spectrum of Harper operator}

Using the characterization in the previous section, it is easy to derive properties on the persistence of the SNA with respect to perturbations in the parameters $a$ and $b$.

It is known that the measure of the spectrum $\Sigma_{b, \omega}$ is given by the formula $|4-2| b||{ }^{39}$ which shows that, when $|b|>2$, SNA occupy a set of parameters $a$ of positive measure. However, $\Sigma_{b, \omega}$ has also been shown to be a Cantor set (this is the "Ten Martini problem"16-18) for irrational $\omega$ and $b \neq 0$, and thus it contains no open intervals. Thus, although the set $\Sigma_{b, \omega}$ of parameters $a$ for which SNA exists is large in the measure-theoretic sense, it is small in the topological sense. In summary, SNA are typical but not robust in the family of Harper maps.

With these arguments we are done with the proof of the Main Theorem.

Remark 4: Note that, although the spectrum is a Cantor set for each $b$, one can find analytic curves in the $(a, b)$ plane where SNA exist for all values in these curves. A trivial example is $a=0$ and $|b|>2, \omega$ irrational, due to the symmetry of the spectrum. Indeed, as it was proved in Ref. 40, there exist analytic curves when $\omega$ is Diophantine which lie entirely in the spectrum for $|b|$ large enough.

Remark 5: One may wonder about the existence of families of linear skew products with persistent nonuniformly hyperbolic behavior. This would imply that the corresponding projectivizations have robust SNA. This question was answered positively by Herman, ${ }^{37}$ considering homotopically nontrivial linear skew products and using simple topological arguments. Other robust SNA have been proved to exist in homotopically nontrivial systems using similar arguments. 3,41

\section{FURTHER PROPERTIES AND EXTENSIONS}

\section{A. Some numerical examples}

As an illustration of the above rigorous results, we performed several numerical computations. In the following, we choose the irrational frequency $\omega=e / 4, b=3$, and we considered $a$ as a moving parameter. The averaged Lyapunov exponent as a function of $a$ is displayed in Fig. 1. Notice that (16) implies that $\bar{\lambda}_{a} \geqslant \log 3 / 2$, so that the Harper cocycle is hyperbolic for all the values $a$. Moreover, the equality holds only if the cocycle is nonuniformly hyperbolic. As a result, the values of $a$ for which $\Phi^{u}$ is a SNA of the Harper map correspond to the "flat pieces" of the graph in Fig. 1, which lie in a Cantor set of measure 2. The "bumps" appear in gaps of the spectrum, that is energies $a$ in the resolvent set, for 


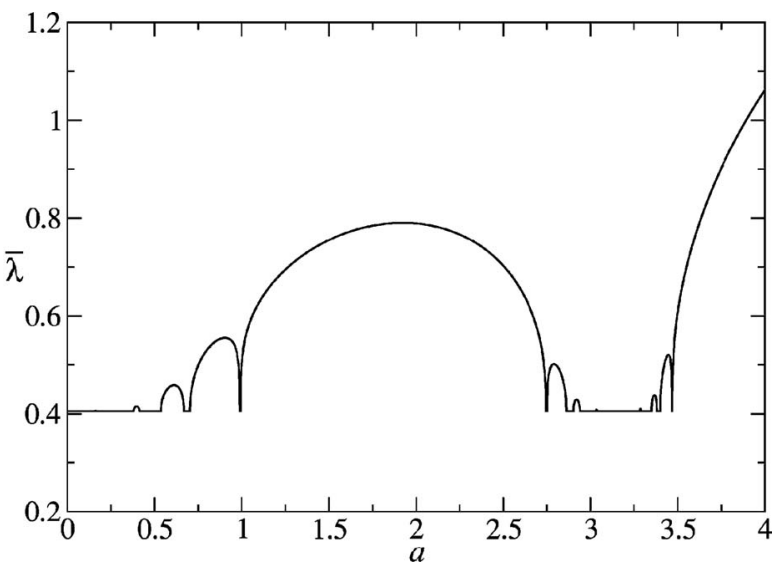

FIG. 1. The averaged Lyapunov exponent $\bar{\lambda}$ of the Harper linear skew product for $b=3$. The Lyapunov exponent of the corresponding attractor of the Harper map is $\beta=-2 \bar{\lambda}$, hence negative. In the spectrum the Lyapunov exponent takes the constant value $\log 3 / 2$. When $a$ approaches the endpoint of a gap, the Lyapunov exponent behaves as a square root, see Ref. 46. This scaling of the Lyapunov exponent seems to be universal (Ref. 20) and has also been observed in the linearization around an attracting invariant curve of a quasiperiodically forced Hénon map. which $\Phi^{u}$ is a continuous attracting invariant curve. We also selected several values of $a$ and computed the attractor $\Phi^{u}$ and the repellor $\Phi^{s}$ of the Harper map for several values of $a$. The results are displayed in Figs. 2 and 3.

Remark 6: Most of the authors in the literature choose the golden mean $\frac{1}{2}(\sqrt{5}-1)$ as the frequency, which has very specific arithmetical properties. In particular it has a periodic (in fact, constant) continuous fraction expansion which makes it convenient for renormalization procedures. The relative distance of $\omega=e / 4$ to the golden mean is less than $10 \%$, but their arithmetical properties are very different. Our choice aims to emphasize that the results presented here are independent of the arithmetical properties of the frequency. 19,20

\section{B. A topological argument for the existence of SNA}

When $\Phi^{u}$ is a continuous attracting invariant curve, and thus $a$ is in a gap of the spectrum, there is a topological index which counts its winding around $\mathbb{P}$, which must coincide with that of $\Phi^{s}$. This winding number changes from gap to
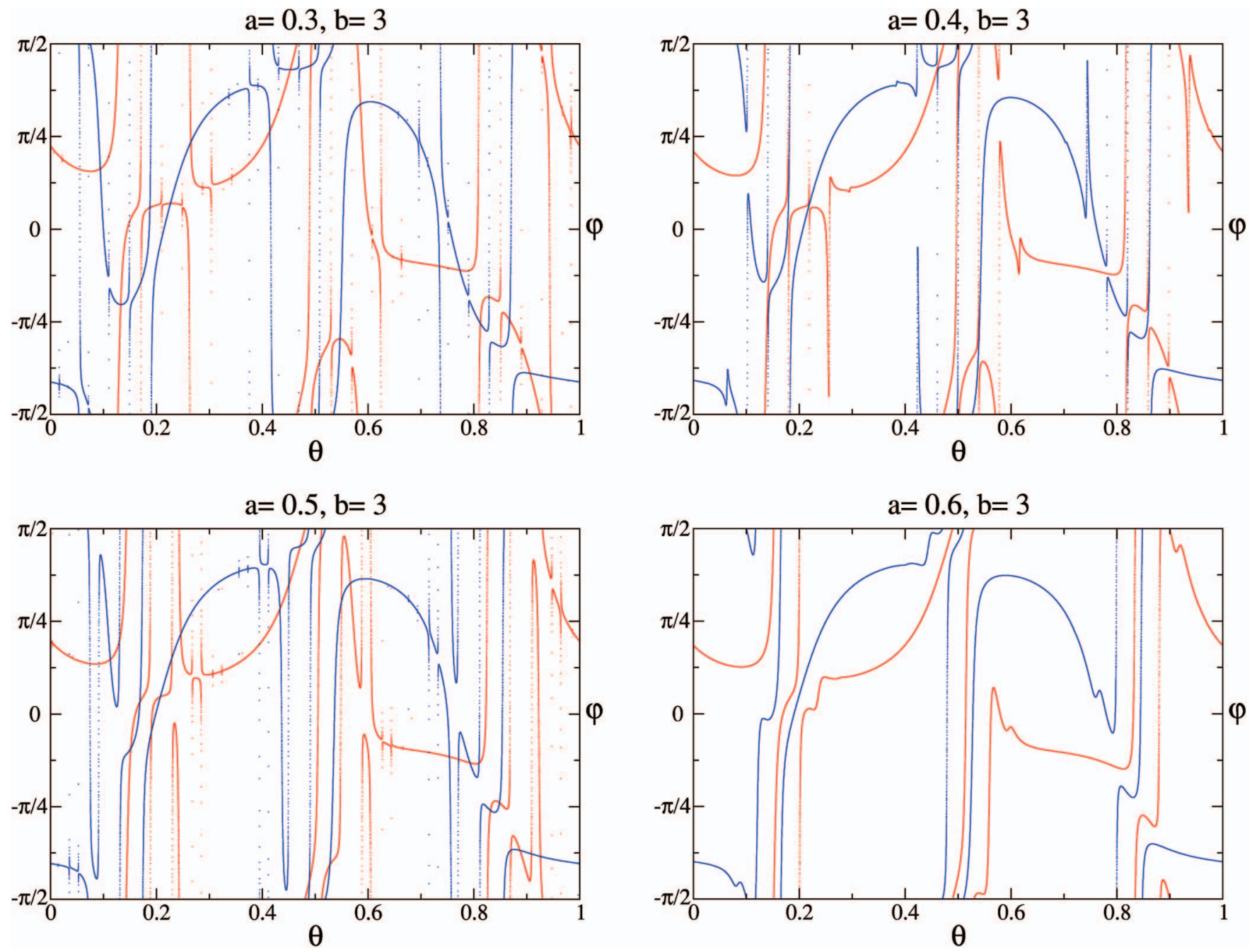

FIG. 2. (Color) The attractor $\Phi^{u}$ and the repellor $\Phi^{s}$ of the Harper map for $a=0.3,0.5$ (SNA) and $a=0.4,0.6$ (continuous invariant curves). Notice that $a$ $=0.4$ and $a=0.6$ correspond to different gaps labelled by the indices of the continuous curves 8 and 5 , respectively. 

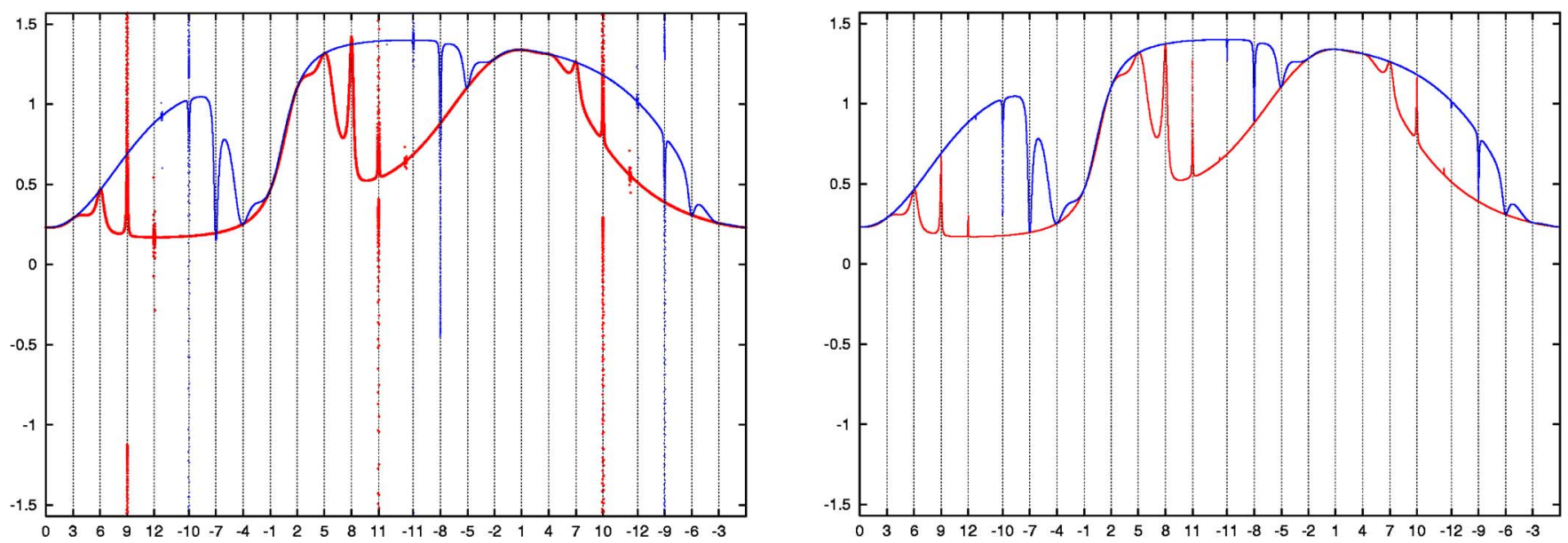

FIG. 3. The attractor $\Phi^{u}$ and the repellor $\Phi^{s}$ of the Harper map for $a=3.47030$ (SNA, left) and $a=3.47031$ (continuous invariant curves, right). Notice the dramatic change in the dynamics with a very small perturbation of $a$. The value $a=3.47031$ belongs to the right-most gap, whose index is 0 (see Fig. 1 ). When $a$ reaches the endpoint of the gap, a localized solution exists and it is supported on the points $\theta=k \omega$, indicated by vertical lines in the figure. Immediately before the collapse (see the picture on the right), we observe that the distance between the two invariant curves attains its minimal value at these vertical lines.

gap, as it can be seen in Figs. 2 and 3 and can be related to the rotation number of (4). ${ }^{42}$ This implies that a continuous attracting invariant curve cannot be continuously deformed from one gap to another, and this shows that in between there must be values of $a$ for which there is no continuous attracting invariant curve. In combination with the positivity of the Lyapunov exponent, this provides another argument for the existence of SNA, although the spectral argument in the previous section yields to quantitative arguments on their abundance.

Inside the gap, the invariant curves $\Phi^{u}$ and $\Phi^{s}$ cannot touch due to the uniform hyperbolicity. However, as the spectral parameter $a$ approaches the endpoint of the gap, they seem to touch, see Fig. 4 which displays the distance between the curves inside the gaps. However, $\varphi^{u}(\theta)$ for a.e. $\theta$, converges to the strange nonchaotic attractor formed at the endpoint of a gap. ${ }^{43}$ To see why this happens, it is worthwhile to recall that inside the gap the elements $\varphi^{u}(\theta)$ and

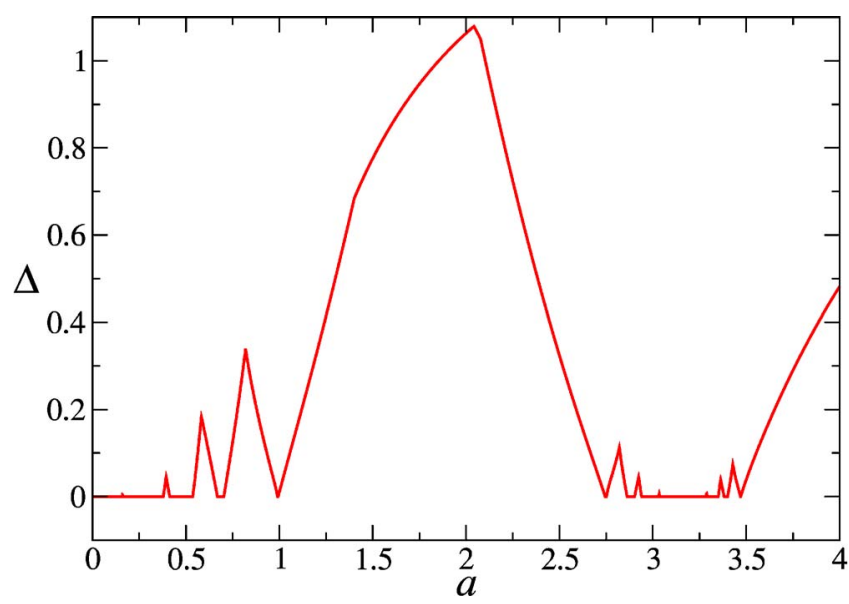

FIG. 4. The minimal distance $\Delta$ between the stable and unstable curves in the Harper map with $b=3$. In the spectrum, the distance is zero. Inside the gaps, the two curves are separated one from each other and the distance is strictly positive. When $a$ approaches the endpoint of a gap, the distance seems to tend to zero in a linear way (Ref. 20). $\varphi^{s}(\theta)$, for each fixed $\theta$, move monotonically in opposite directions as the spectral parameter increases. ${ }^{44}$

\section{SNA and localization}

In this paper we have proved the existence of SNA in Harper maps with $\omega$ irrational, $|b|>2$ and $a$ in the spectrum without resorting to the possible localization properties of the spectral problem. Recall that $a$ is a point eigenvalue of a Harper operator $H_{b, \omega, \theta_{0}}$ if the corresponding eigenvalue (Harper) equation has a nontrivial localized solution $\psi$ $=\left(\psi_{n}\right)_{n \in Z}$ which is square integrable or even decays exponentially with $|n|$. If the set of localized eigenvectors of a Harper operator forms a complete orthogonal basis of $\ell^{2}(\mathbb{Z})$ then the spectrum is pure point.

In previous work on the existence of SNA in Harper maps, localization was seen as a justification for the strangeness of SNA, in the regime of nonzero Lyapunov exponents. ${ }^{10,11,14,21,22}$ As we have seen, we do not use localization to prove the existence of SNA. Besides, localization may not hold in all the Harper maps considered here, because an energy $a$ in the spectrum with nonzero Lyapunov exponent (for which the Harper map has a SNA) may not be an eigenvalue of the operator. Indeed, if $\omega$ is not Diophantine, localization may only hold for $|b|>2$ large enough (depending on $\omega) .{ }^{18}$ Even in the Diophantine case, the spectrum also contains a residual set of energies which are not point eigenvalues. ${ }^{45,46}$

Nevertheless, exponentially localized solutions, whenever they exist, are useful for the description of many properties of SNA in Harper maps, since they can be seen as heteroclinic solutions which connect $\Phi^{s}$ (when $n \rightarrow-\infty$ ) and $\Phi^{u}$ (when $n \rightarrow+\infty$ ). Moreover, whenever an exponentially localized solution exists at the endpoint of a gap (this happens, for instance, when $\omega$ is Diophantine and $|b|$ is large), simple duality arguments ${ }^{46}$ show that the SNA must be discontinuous at the points $\theta=k \omega$, with $k \in \mathbb{Z}$. For an illustration, see Fig. 3. 


\section{Extension to other families of maps}

We would like to emphasize that the arguments presented in this paper are based on the theory of nonuniformly hyperbolic dynamical systems (Pesin theory) and thus they provide a fertile ground for extensions to other interesting models. Here we present two systems where the generalization is straightforward.

In combination results in the spectral theory of quasiperiodic Schrödinger operators, we can also show the existence and abundance of SNA in several families of Harpertype maps

$$
\begin{aligned}
y_{n+1} & =\frac{1}{a-b V\left(2 \pi \theta_{n}\right)-y_{n}}, \\
\theta_{n+1} & =\theta_{n}+\omega \quad(\bmod 1),
\end{aligned}
$$

where $V: T \rightarrow R$ is a nonconstant real analytic function and $\omega$ is an irrational number. All the arguments of nonuniform hyperbolicity also apply to this case and to show the existence of SNA one only needs positivity of the Lyapunov exponent in the spectrum. This is granted by Ref. 47, where it is shown that the Lyapunov exponent is positive for all $a$ $\in \mathbb{R}$ as long as $b$ is greater than some constant depending only on $V$. Extensions to more frequencies also hold (see Ref. 48, and references therein) for different results on positivity of the Lyapunov exponent in other models, that are not necessarily quasiperiodic.

Another easy translation of our results is in the following class of differential equations, where virtually all the same techniques apply (see Ref. 15 for a very similar equation). Consider the flow on $\mathbb{T} \times \mathbb{T}^{2}$ given by the equations

$$
\begin{aligned}
& \varphi^{\prime}=\left(a+b\left(\cos \theta_{1}+\cos \theta_{2}\right)\right) \sin ^{2} \varphi+\cos ^{2} \varphi, \\
& \theta_{1}^{\prime}=1, \quad \theta_{2}^{\prime}=\omega,
\end{aligned}
$$

where $\omega$ is an irrational number and $\theta_{1}, \theta_{2} \in \mathbb{T}$. Since this is the projectivization of the equation

$$
x^{\prime \prime}+\left(a+b\left(\cos \theta_{1}+\cos \theta_{2}\right)\right) x=0, \quad \theta_{1}^{\prime}=1, \quad \theta_{2}^{\prime}=\omega,
$$

expressed in polar coordinates (the eigenvalue equation of a continuous quasiperiodic Schrödinger operator), all the arguments can be used to derive the existence of SNA if we are able to prove positivity of the Lyapunov exponent in the spectrum. This was done again, in Ref. 47, where it was shown that the Lyapunov exponent is positive when $|b|$ is large enough in an open set at the bottom of the spectrum. For these values of $a$, there will be a SNA in Eq. (18).

\section{ACKNOWLEDGMENTS}

The authors thank R. de la Llave for encouragement and advice which largely improved the manuscript. A.H. was par- tially supported by MCyT/FEDER Grant No. BFM200307521-C02-01 and CIRIT Grant No. 2001SGR-70. J.P. was partially supported by MCyT/FEDER Grant No. BFM200309504-C02-01 and CIRIT Grant No. 2001SGR-70.

${ }^{1}$ D. Ruelle and F. Takens, Commun. Math. Phys. 20, 167 (1971).

${ }^{2}$ J.-P. Eckmann and D. Ruelle, Rev. Mod. Phys. 57, 617 (1985).

${ }^{3}$ C. Grebogi, E. Ott, S. Pelikan, and J. A. Yorke, Physica D 13, 261 (1984).

${ }^{4}$ A. Prasad, S. S. Negi, and R. Ramaswamy, Int. J. Bifurcation Chaos Appl. Sci. Eng. 11, 291 (2001).

${ }^{5}$ G. Keller, Fund. Math. 151, 139 (1996).

${ }^{6}$ P. Glendinning, Dyn. Syst. 17, 287 (2002).

${ }^{7}$ J. Stark, Dyn. Syst. 18, 351 (2003).

${ }^{8}$ T. Jäger, Ergod. Theory Dyn. Syst.. (to be published).

${ }^{9}$ K. Bjerklov, Ergod. Theory Dyn. Syst. 25, 1015 (2005).

${ }^{10}$ J. A. Ketoja and I. I. Satija, Physica D 109, 70 (1997).

${ }^{11}$ J. A. Ketoja and I. I. Satija, Phys. Rev. Lett. 75, 2762 (1995).

${ }^{12}$ S. S. Singh and R. Ramaswany, Phys. Rev. Lett. 83, 4530 (1999).

${ }^{13}$ S. S. Singh and R. Ramaswany, Phys. Rev. E 64, 045204 (2001).

${ }^{14}$ B. D. Mestel, A. H. Osbaldestin, and B. Winn, J. Math. Phys. 41, 8304 (2000).

${ }^{15}$ A. Bondeson, E. Ott, and T. M. Antonsen, Jr., Phys. Rev. Lett. 55, 2103 (1985).

${ }^{16}$ M. D. Choi, G. A. Elliott, and N. Yui, Invent. Math. 99, 225 (1990).

${ }^{17}$ J. Puig, Commun. Math. Phys. 244, 297 (2004).

${ }^{18}$ A. Avila and S. Jitomirskaya, Ann. Math. (to be published).

${ }^{19} \mathrm{~A}$. Haro and R. de la Llave (preprint, 2005).

${ }^{20}$ A. Haro and R. de la Llave, Chaos 16, 013120 (2006).

${ }^{21}$ B. D. Mestel and A. H. Osbaldestin, J. Math. Phys. 45, 5042 (2004).

${ }^{22}$ B. D. Mestel and A. H. Osbaldestin, J. Phys. A 37, 9071 (2004).

${ }^{23}$ P. Harper, Proc. Phys. Soc. London A68, 874 (1955).

${ }^{24}$ J. B. Sokoloff, Phys. Rep. 126, 189 (1985).

${ }^{25}$ K. v. Klitzing, G. Dorda, and M. Pepper, Phys. Rev. Lett. 45, 494 (1980).

${ }^{26}$ V. I. Oseledec, Trudy Moskov. Mat. Obšč. 19, 179 (1968).

${ }^{27}$ J. F. C. Kingman, J. R. Stat. Soc. Ser. B (Methodol.) 30, 499 (1968).

${ }^{28}$ R. J. Sacker and G. R. Sell, J. Differ. Equations 27, 320 (1978).

${ }^{29}$ J. F. Selgrade, Trans. Am. Math. Soc. 203, 359 (1975).

${ }^{30}$ R. Johnson and G. Sell, J. Differ. Equations 41, 262 (1981).

${ }^{31}$ R. Johnson, J. Differ. Equations 35, 366 (1980).

${ }^{32}$ M. Hirsch, C. Pugh, and M. Shub, Invariant Manifolds, Lecture Notes in Mathematics (Springer-Verlag, Berlin, 1977), Vol. 583.

${ }^{33} \mathrm{~A}$. Haro and R. de la Llave (preprint, 2003).

${ }^{34}$ S. Datta, T. Jäger, G. Keller, and R. Ramaswamy, Nonlinearity 17, 2315 (2004).

${ }^{35}$ R. Mañé, Trans. Am. Math. Soc. 246, 261 (1978).

${ }^{36}$ R. Johnson, J. Differ. Equations 46, 165 (1982).

${ }^{37}$ M. Herman, Comment. Math. Helv. 58, 453 (1983).

${ }^{38}$ J. Bourgain and S. Jitomirskaya, J. Stat. Phys. 108, 1203 (2002).

${ }^{39}$ S. Y. Jitomirskaya and I. V. Krasovsky, Math. Res. Lett. 9, 413 (2002).

${ }^{40}$ J. Puig and C. Simó, Ergod. Theory Dyn. Syst. 26, 481 (2006).

${ }^{41}$ B. Hunt, and E. Ott, Phys. Rev. Lett. 87, 254101 (2001).

${ }^{42}$ R. Johnson and J. Moser, Commun. Math. Phys. 84, 403 (1982).

${ }^{43}$ R. Johnson, Ill. J. Math. 28, 397 (1984).

${ }^{44}$ E. Coddington and N. Levinson, Theory of Ordinary Differential Equations (McGraw-Hill, New York, 1955).

${ }^{45}$ S. Jitomirskaya and B. Simon, Commun. Math. Phys. 165, 201 (1994).

${ }^{46}$ J. Puig, Nonlinearity 19, 355 (2006).

${ }^{47}$ E. Sorets and T. Spencer, Commun. Math. Phys. 142, 543 (1991).

${ }^{48} \mathrm{~J}$. Bourgain, Green's Function Estimates for Lattice Schrödinger Operators and Applications, Annals of Mathematics Studies (Princeton University Press, Princeton, 2005). 